\title{
THE ELASTIC FIELDS OF A DISLOCATION IN AN ANISOTROPIC STRIP
}

\author{
KUANG-CHONG WU and YU-TSUNG CHIU \\ Institute of Applied Mechanics, National Taiwan University, Taipei 106, Taiwan
}

(Received 7 June 1994)

\begin{abstract}
The elastic fields of a dislocation in a general anisotropic strip are analysed. The analysis is made by using the Stroh formalism for anisotropic elasticity in conjunction with the Fourier transform. The surfaces of the strip are considered either free or fixed. The image forces on an edge dislocation in tungsten and potassium are computed as functions of the position of the dislocation and the inclination of the slip planes. The interactive forces between two like dislocations on the same slip plane are also calculated.
\end{abstract}

\section{INTRODUCTION}

The motion of a dislocation in an elastic material is related to the energetic force acting on the dislocation. In an unbounded homogeneous medium without external applied stress, the force is caused only by other defects. In real materials of finite extent, however, image forces due to the presence of boundaries must also be considered.

The simplest example of the image force induced by boundaries is provided by a dislocation in a half-space. For isotropic half-spaces, the expression for the force is given by Head (1953) for screw dislocations and by Dundurs and Sendeckyj (1965) for edge dislocations. A similar expression valid for a dislocation with arbitrary Burgers vector in an anisotropic half-space is obtained by Barnett and Lothe (1974). The aforementioned works actually are concerned with a bimaterial composite of which a semi-infinite medium is a special case. The more complicated example is supplied by a dislocation in an infinite strip. The stress field of an edge dislocation in an isotropic layer on an isotropic substrate with Burgers vector normal to the interface was analysed by Lee and Dundurs (1973). By letting the substrate be infinitely soft or hard, Lee and Dundurs also investigated the cases of a free layer and free fixed layer. The problem of an edge dislocation in a free isotropic layer with Burgers vector parallel to the surfaces was studied by Nabarro and Kostlan (1978) and independently by Moss and Hoover (1978). A similar problem for an anisotropic strip with free surfaces was investigated by Chou (1963). Chou's treatment is limited to orthotropic materials with one of the symmetry planes coinciding with the plane of the strip.

In this paper the elastic fields of a dislocation in a general anisotropic strip are presented. The boundary conditions of the strip surfaces are either free or fixed. The solution procedure is based on a synthesis of Stroh's formalism (Stroh, 1958, 1962) for anisotropic elasticity and the Fourier transform. The elastic field of interest is divided into a singular part and a regular part. The singular part is the field due to a dislocation in an unbounded medium and the regular part is obtained by annihilating the tractions or displacements of the singular field on the strip surfaces. The image forces are computed as a function of the position of the dislocation and the inclination of the slip plane for isotropic tungsten (W), and anisotropic potassium (K). Interactive forces between two like dislocations on the central plane of the strip in these materials are also calculated to study the influence of the boundaries.

The paper is arranged as follows. First Stroh's formalism is outlined in section 2. The Fourier transform method synthesized with Stroh's formalism is introduced in section 3. The elastic fields of a dislocation in an anisotropic strip are derived in section 4 . Results of 
image forces of a dislocation and interactive forces of two similar dislocations are presented in Section 5.

\section{STROH'S FORMALISM}

In this section, a brief introduction to Stroh's formulation for anisotropic elasticity is given.

For two-dimensional deformations dependent only on $x_{1}$ and $x_{2}$, the general expressions for displacement $\mathbf{u}$ and stress function $\phi$ can be written as

$$
\begin{aligned}
\mathbf{u} & =\mathbf{A f}+\overline{\mathbf{A}} \overline{\mathbf{f}} \\
\phi & =\mathbf{B} \mathbf{f}+\overline{\mathbf{B}} \overline{\mathbf{f}}
\end{aligned}
$$

where $\mathbf{f}=\left\{f_{1}\left(z_{1}\right), f_{2}\left(z_{2}\right), f_{3}\left(z_{3}\right)\right\}^{\mathrm{T}}$ with $z_{\alpha}=x_{1}+p_{\alpha} x_{2}$ and the overline denotes a complex conjugate. The complex constants $p_{\alpha}$ and the complex matrices $\mathbf{A}$ and $\mathbf{B}$ are functions of elastic constants. Without loss of generality, the imaginary part of $p_{\alpha}$ is taken to be positive. The stresses are related to the stress function $\phi$ by

$$
\sigma_{i 1}=-\frac{\partial \phi_{i}}{\partial x_{2}}, \quad \sigma_{i 2}=\frac{\partial \phi_{i}}{\partial x_{1}}, \quad i=1,2,3
$$

With a six-by-six matrix $\mathbf{U}$ defined as

$$
\mathbf{U}=\left[\begin{array}{cc}
\mathbf{A} & \overline{\mathbf{A}} \\
\mathbf{B} & \overline{\mathbf{B}}
\end{array}\right]
$$

eqn (1) can be expressed as

$$
\mathbf{v} \equiv\left\{\begin{array}{l}
\mathbf{u} \\
\phi
\end{array}\right\}=\mathbf{U}\left\{\begin{array}{l}
\mathbf{f} \\
\mathbf{f}
\end{array}\right\}
$$

The matrix $\mathbf{U}$ has the following orthogonality relations:

$$
\begin{aligned}
& \mathbf{U}^{\mathrm{T}} \mathbf{J} \mathbf{U}=\hat{\mathbf{I}} \\
& \mathbf{J} \mathbf{U}^{\mathrm{T}}=\hat{\mathbf{I}} \\
& \mathbf{U} \mathbf{U}^{\mathrm{T}} \mathbf{J}=\hat{\mathbf{I}},
\end{aligned}
$$

where $\mathbf{I}$ is the six-by-six identity matrix and $\mathbf{J}$ is defined as

$$
\mathbf{J}=\left[\begin{array}{ll}
\mathbf{0} & \mathbf{I} \\
\mathbf{I} & \mathbf{0}
\end{array}\right]
$$

In the above, $\mathbf{I}$ is the three-by-three identity matrix. For future reference, two real matrices $\mathbf{L}$ and $\mathbf{H}$ are defined here as

$$
\begin{gathered}
\mathbf{L}=-2 i \mathbf{B B}^{\mathrm{T}} \\
\mathbf{H}=2 i \mathbf{A} \mathbf{A}^{\mathrm{T}} .
\end{gathered}
$$

In the above expressions, the complex constants $p_{\alpha}$ are assumed to be distinct. Consequently, Stroh's formalism cannot be directly applied to isotropic materials for which $p=i$ is a double root for plane strain deformation. However, in many problems, including the 
one considered in this paper, results for isotropic materials can be obtained by a limiting process discussed in Wu (1991).

\section{FOURIER TRANSFORM}

Let $\tilde{\mathbf{f}}$ be the Fourier transform of the Stroh function $\mathbf{f}$ given by

$$
\tilde{\mathbf{f}}\left(\omega, x_{2}\right)=\int_{-\infty}^{\infty} \mathbf{f}\left(x_{1}, x_{2}\right) \mathrm{e}^{\mathrm{i} \omega x_{1}} \mathrm{~d} x_{1}
$$

The function $\mathbf{f}$ can be obtained from the inverse Fourier transform of $\tilde{\mathbf{f}}$ as

$$
\mathbf{f}\left(x_{1}, x_{2}\right)=\frac{1}{2 \pi} \int_{-\infty}^{\infty} \tilde{\mathbf{f}}\left(\omega, x_{2}\right) \mathrm{e}^{-i \omega x_{1}} \mathrm{~d} \omega
$$

With eqn (7), the relation (3) in the Fourier transform domain is

$$
\mathbf{v} \equiv\left\{\begin{array}{l}
\tilde{\mathbf{u}} \\
\tilde{\phi}
\end{array}\right\}=\mathbf{U}\left\{\begin{array}{c}
\tilde{f}\left(\omega, x_{2}\right) \\
\tilde{\tilde{f}}\left(-\omega, x_{2}\right)
\end{array}\right\}
$$

in which $\tilde{\mathbf{u}}\left(\omega, x_{2}\right)$ and $\tilde{\phi}\left(\omega, x_{2}\right)$ are the Fourier transforms of Stroh's displacement vector and stress function vector, respectively.

Since $\mathbf{f}$ is an analytic function of $z_{\alpha}$, eqn (8) implies that $\tilde{f}$ must be in the form

$$
\tilde{\mathbf{f}}\left(\omega, x_{2}\right)=\left\langle\mathrm{e}^{-\mathrm{i} \omega p x_{2}}\right\rangle \tilde{\mathbf{h}}(\omega),
$$

where $\widetilde{\mathbf{h}}(\omega)$ is an arbitrary function of $\omega$ and $\left\langle\mathrm{e}^{-\mathrm{i} \omega p x_{2}}\right\rangle$ stands for a three-by-three diagonal matrix with $\mathrm{e}^{-\mathrm{i} \omega p_{\alpha} x_{2}}, \alpha=1,2,3$, as the diagonal elements. Let $\widetilde{\mathbf{d}}(\omega)$ and $\tilde{\mathbf{s}}(\omega)$ be defined as

$$
\left\{\begin{array}{l}
\tilde{\mathbf{d}}(\omega) \\
\tilde{\mathbf{s}}(\omega)
\end{array}\right\}=\mathbf{U}\left\{\begin{array}{l}
\tilde{\mathbf{h}}(\omega) \\
\tilde{\tilde{\mathbf{h}}}(-\omega)
\end{array}\right\}
$$

With eqns (11) and (4), the function $\tilde{\mathbf{f}}$ can be represented as

$$
\left\{\begin{array}{l}
\tilde{\mathbf{f}}\left(\omega, x_{2}\right) \\
\overline{\tilde{\mathbf{f}}}\left(-\omega, x_{2}\right)
\end{array}\right\}=\tilde{\boldsymbol{\Lambda}}\left(\omega, x_{2}\right) \mathbf{U}^{\mathrm{T}} \mathbf{J}\left\{\begin{array}{l}
\tilde{\mathbf{d}}(\omega) \\
\tilde{\mathbf{s}}(\omega)
\end{array}\right\},
$$

where $\tilde{\mathbf{\Lambda}}\left(\omega, x_{2}\right)$ is a six-by-six matrix function defined as

$$
\tilde{\boldsymbol{\Lambda}}\left(\omega, x_{2}\right)=\left[\begin{array}{cc}
\left\langle\mathrm{e}^{-\mathrm{i} \omega p x_{2}}\right\rangle & \mathbf{0} \\
0 & \left\langle\mathrm{e}^{-\mathrm{i} \omega \overline{\bar{p} x_{2}}}\right\rangle
\end{array}\right] .
$$

Substituting eqn (12) into eqn (9), we have

$$
\tilde{\mathbf{v}}\left(\omega, x_{2}\right)=\widetilde{\mathbf{G}}\left(\omega, x_{2}\right)\left\{\begin{array}{l}
\tilde{\mathbf{d}}(\omega) \\
\tilde{\mathbf{s}}(\omega)
\end{array}\right\} .
$$

The matrix $\tilde{\mathbf{G}}\left(\omega, x_{2}\right)$ in eqn (13) is defined as

$$
\tilde{\mathbf{G}}\left(\omega, x_{2}\right)=\mathbf{U} \tilde{\mathbf{\Lambda}}\left(\omega, x_{2}\right) \mathbf{U}^{\mathrm{T}} \mathbf{J} .
$$

The matrix $\tilde{\mathbf{G}}\left(\omega, x_{2}\right)$ can be divided into four submatrices as follows: 


$$
\tilde{\mathbf{G}}\left(\omega, x_{2}\right)=\left[\begin{array}{ll}
\tilde{\mathbf{G}}_{1}\left(\omega, x_{2}\right) & \tilde{\mathbf{G}}_{2}\left(\omega, x_{2}\right) \\
\widetilde{\mathbf{G}}_{3}\left(\omega, x_{2}\right) & \widetilde{\mathbf{G}}_{1}^{\mathrm{T}}\left(\omega, x_{2}\right)
\end{array}\right],
$$

where

$$
\begin{aligned}
& \widetilde{\mathbf{G}}_{1}\left(\omega, x_{2}\right)=\mathbf{A}\left\langle\mathrm{e}^{-1 \omega p x_{2}}\right\rangle \mathbf{B}^{\mathrm{T}}+\overline{\mathbf{A}}\left\langle\mathrm{e}^{-\mathrm{i} \omega \bar{p} x_{2}}\right\rangle \overline{\mathbf{B}}^{\mathrm{T}} \\
& \widetilde{\mathbf{G}}_{2}\left(\omega, x_{2}\right)=\mathbf{A}\left\langle\mathrm{e}^{-\mathrm{i} \omega p x_{2}}\right\rangle \mathbf{A}^{\mathrm{T}}+\overline{\mathbf{A}}\left\langle\mathrm{e}^{-i \omega \bar{p} x_{2}}\right\rangle \overline{\mathbf{A}}^{\mathrm{T}} \\
& \widetilde{\mathbf{G}}_{3}\left(\omega, x_{2}\right)=\mathbf{B}\left\langle\mathrm{e}^{-1 \omega p x_{2}}\right\rangle \mathbf{B}^{\mathrm{T}}+\overline{\mathbf{B}}\left\langle\mathrm{e}^{-\mathrm{i} \omega \bar{p} x_{2}}\right\rangle \overline{\mathbf{B}}^{\mathrm{T}} .
\end{aligned}
$$

The three submatrices play an important role in Wang et al. (1991) concerning an anisotropic semi-infinite strip. It appears that these matrices are inherited in problems related to strips. Because of the orthogonality relations of eqn (4), the matrix $\widetilde{\mathbf{G}}\left(\omega, x_{2}\right)$ has the following very useful properties:

$$
\begin{aligned}
\widetilde{\mathbf{G}}(\omega, 0) & =\mathbf{I} \\
\widetilde{\mathbf{G}}\left(\omega, x_{2}\right) & =\widetilde{\mathbf{G}}\left(\omega, x_{2}-c\right) \widetilde{\mathbf{G}}(\omega, c) \\
\widetilde{\mathbf{G}}\left(\omega,-x_{2}\right) & =\left[\widetilde{\mathbf{G}}\left(\omega, x_{2}\right)\right]^{-1}
\end{aligned}
$$

where $c$ is an arbitrary constant.

\section{ELASTIC FIELDS}

In this section, the elastic fields due to a dislocation with Burgers vector $\mathbf{b}$ in an infinite strip of thickness $2 a$ are derived. The $\left(x_{1}, x_{3}\right)$ plane is taken as the central plane of the strip. The dislocation line is along the $x_{3}$ axis, piercing the $\left(x_{1}, x_{2}\right)$ plane at $(0, \eta a)$. The boundary conditions on the faces are assumed to be either free or fixed. The configuration is shown in Fig. 1. Only the elastic fields for $a=1$ are derived here. Let the Stroh function for the case $a=1$ be $\mathbf{f}\left(x_{1}, x_{2}\right)$ and the corresponding displacement, stress function and stress be $\mathbf{u}\left(x_{1}, x_{2}\right), \phi\left(x_{1}, x_{2}\right)$ and $\sigma\left(x_{1}, x_{2}\right)$, respectively. The Stroh function, displacement, stress function and stress for arbitrary $a$ are simply $\mathbf{f}\left(x_{1} / a, x_{2} / a\right), \mathbf{u}\left(x_{1} / a, x_{2} / a\right), \phi\left(x_{1} / a, x_{2} / a\right)$ and $\sigma\left(x_{1} / a, x_{2} / a\right) / a$, respectively.

Let the solution of the problem of interest in the transform domain be expressed as

$$
\tilde{\mathbf{v}}\left(\omega, x_{2}\right)=\tilde{\mathbf{v}}_{\omega \infty}\left(\omega, x_{2} ; \eta\right)+\tilde{\mathbf{v}}_{1 \mathrm{~m}}\left(\omega, x_{2}\right)
$$

In eqn (15), $\tilde{\mathbf{v}}_{\infty}\left(\omega, x_{2}\right)$ is the Fourier transform of the elastic field for a dislocation in an unbounded body of the same material and $\tilde{v}_{\text {im }}\left(\omega, x_{2}\right)$ is the Fourier transform of the image field due to the presence of the boundaries.

The Stroh function for $\mathbf{v}_{\infty}$ is given by (Stroh, 1958)

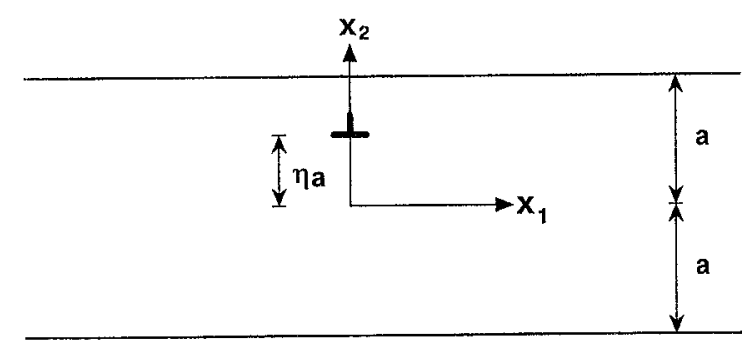

Fig. 1. A dislocation in an elastic strip of thickness $2 a$. 


$$
\mathbf{f}_{\infty}(z)=\frac{1}{2 \pi i}\langle\log (z-\zeta)\rangle \mathbf{B}^{\mathrm{T}} \mathbf{b}
$$

where $\zeta_{\alpha}=p_{\alpha} \eta, \alpha=1,2,3$. Since $\mathbf{f}_{\infty}$ is unbounded as $x_{1} \rightarrow \infty, \tilde{\mathbf{f}}_{\infty}$ defined by eqn (7) does not exist in the ordinary sense. We shall regard $\mathbf{f}_{\infty}$ as

$$
\tilde{\mathbf{f}}_{\infty}=-\frac{\tilde{\mathbf{f}}_{\infty}^{\prime}}{i \omega}
$$

where $\tilde{\mathbf{f}}_{\infty}^{\prime}$ is the Fourier transform of $\mathbf{f}_{\infty}^{\prime}$ defined as

$$
\mathbf{f}_{\infty}^{\prime}(z) \equiv \frac{\partial \mathbf{f}_{\infty}}{\partial x_{1}}=\frac{1}{2 \pi i}\left\langle\frac{1}{z-\zeta}\right\rangle \mathbf{B}^{\mathbf{T}} \mathbf{b}
$$

It can be shown that $\tilde{\mathbf{f}}_{\infty}^{\prime}$ is given by

$$
\tilde{\mathbf{f}}_{\infty}^{\prime}=-\operatorname{sgn}(y) H(-\operatorname{sgn}(y) \omega)\left\langle\mathrm{e}^{-\mathrm{i} \omega p y}\right\rangle \mathbf{B}^{\mathrm{T}} \mathbf{b},
$$

where $y=x_{2}-\eta, H(\omega)$ is the Heaviside function and $\operatorname{sgn}(y)$ is the sign function defined as

$$
\begin{gathered}
H(\omega)= \begin{cases}1, & \omega>0 \\
0, & \omega<0\end{cases} \\
\operatorname{sgn}(y)=\left\{\begin{aligned}
1, & y>0 \\
-1, & y<0
\end{aligned}\right.
\end{gathered}
$$

By substituting eqn (19) into eqn (17) and eqn (17) into eqn (9), the corresponding $\tilde{\mathbf{v}}_{\infty}$ is obtained as

$$
\tilde{\mathbf{v}}_{\infty}\left(\omega, x_{2} ; \eta\right)=\frac{1}{i \omega} \mathbf{U} \mathbf{H}_{\mathrm{V}}(\omega, y) \tilde{\mathbf{\Lambda}}(\omega, y) \mathbf{U}^{\mathrm{T}} \mathbf{J g}
$$

where the matrix function $\mathbf{H}_{\mathrm{v}}(\omega, Y)$ and the vector $\mathbf{g}$ are given by

$$
\begin{aligned}
\mathbf{H}_{\mathrm{v}}(\omega, y) & =\operatorname{sgn}(y)\left[\begin{array}{cc}
H(-\operatorname{sgn}(y) \omega) \mathbf{I} & \mathbf{0} \\
\mathbf{0} & H(\operatorname{sgn}(y) \omega) \mathbf{I}
\end{array}\right] \\
\mathbf{g} & =\left\{\begin{array}{l}
\mathbf{b} \\
\mathbf{0}
\end{array}\right\} .
\end{aligned}
$$

For convenience let $\tilde{\mathbf{v}}_{\mathrm{im}}\left(\omega, x_{2}\right)$ be expressed as

$$
\tilde{\mathbf{v}}_{\mathrm{im}}\left(\omega, x_{2}\right)=\tilde{\mathbf{G}}\left(\omega, x_{2}+1\right)\left\{\begin{array}{c}
\tilde{\mathbf{d}}_{0}(\omega) \\
\tilde{\mathbf{s}}_{0}(\omega)
\end{array}\right\}+\tilde{\mathbf{G}}\left(\omega, x_{2}-1\right)\left\{\begin{array}{c}
\tilde{\mathbf{d}}_{1}(\omega) \\
\tilde{\mathbf{s}}_{1}(\omega)
\end{array}\right\},
$$

where $\widetilde{\mathbf{d}}_{0}, \widetilde{\mathbf{s}}_{0}, \widetilde{\mathbf{d}}_{1}$ and $\widetilde{\mathbf{s}}_{1}$ are unknowns to be determined from the boundary conditions. At the face $x_{2}=1$, eqns (23) and (15) yield

$$
\tilde{\mathbf{v}}(\omega, 1)=\left\{\begin{array}{l}
\tilde{\mathbf{u}}_{\infty}(\omega, 1 ; \eta) \\
\tilde{\phi}_{\infty}(\omega, 1 ; \eta)
\end{array}\right\}+\widetilde{\mathbf{G}}(\omega, 2)\left\{\begin{array}{l}
\tilde{\mathbf{d}}_{0}(\omega) \\
\tilde{\mathbf{s}}_{0}(\omega)
\end{array}\right\}+\left\{\begin{array}{l}
\tilde{\mathbf{d}}_{1}(\omega) \\
\tilde{\mathbf{s}}_{1}(\omega)
\end{array}\right\},
$$

and at $x_{2}=-1$, 


$$
\tilde{\mathbf{v}}(\omega,-1)=\left\{\begin{array}{l}
\tilde{\mathbf{u}}_{\infty}(\omega,-1 ; \eta) \\
\tilde{\phi}_{\infty}(\omega,-1 ; \eta)
\end{array}\right\}+\left\{\begin{array}{l}
\tilde{\mathbf{d}}_{0}(\omega) \\
\tilde{\mathbf{s}}_{0}(\omega)
\end{array}\right\}+\tilde{\mathbf{G}}(\omega,-2)\left\{\begin{array}{l}
\tilde{\mathbf{d}}_{1}(\omega) \\
\tilde{\mathbf{s}}_{1}(\omega)
\end{array}\right\}
$$

where eqn (14) has been used. Consider the following three cases of boundary conditions: (1) $\tilde{\phi}=0$ at $x_{2}= \pm 1$; (2) $\tilde{\mathbf{u}}=0$ at $x_{2}=-1$ and $\tilde{\phi}=0$ at $x_{2}=1$; (3) $\tilde{\mathbf{u}}=0$ at $x_{2}= \pm 1$. For case (1), let $\tilde{\mathbf{s}}_{0}=\tilde{\mathbf{s}}_{1}=0$ identically. The remaining unknowns $\tilde{\mathbf{d}}_{0}$ and $\tilde{\mathbf{d}}_{1}$ can be determined from eqns (24) and (25) as

$$
\begin{aligned}
\tilde{\mathbf{d}}_{1}(\omega) & =-\left[\widetilde{\mathbf{G}}_{3}(\omega,-2)\right]^{-1}\left\{\tilde{\phi}_{\infty}(\omega,-1 ; \eta)\right\} \\
\tilde{\mathbf{d}}_{0}(\omega) & =-\left[\widetilde{\mathbf{G}}_{3}(\omega, 2)\right]^{-1}\left\{\tilde{\phi}_{\infty}(\omega, 1 ; \eta)\right\} .
\end{aligned}
$$

For case (2), setting $\widetilde{\mathbf{d}}_{0}=\tilde{\mathbf{s}}_{1}=0$, eqns (24) and (25) lead to

$$
\begin{aligned}
& \widetilde{\mathbf{d}}_{1}(\omega)=-\left[\tilde{\mathbf{G}}_{1}(\omega,-2)\right]^{-1}\left\{\tilde{\mathbf{u}}_{\infty}(\omega,-1 ; \eta)\right\} \\
& \tilde{\mathbf{s}}_{0}(\omega)=-\left[\tilde{\mathbf{G}}_{1}^{\mathrm{T}}(\omega, 2)\right]^{-1}\left\{\tilde{\phi}_{\infty}(\omega, 1 ; \eta)\right\} .
\end{aligned}
$$

Similarly for case (3), $\widetilde{\mathbf{d}}_{0}=\widetilde{\mathbf{d}}_{1}=0$ and

$$
\begin{aligned}
& \tilde{\mathbf{s}}_{1}(\omega)=-\left[\tilde{\mathbf{G}}_{2}(\omega,-2)\right]^{-1}\left\{\tilde{\mathbf{u}}_{\infty}(\omega,-1 ; \eta)\right\} \\
& \tilde{\mathbf{s}}_{0}(\omega)=-\left[\tilde{\mathbf{G}}_{2}(\omega, 2)\right]^{-1}\left\{\tilde{\mathbf{u}}_{\infty}(\omega, 1 ; \eta)\right\} .
\end{aligned}
$$

The image field in the physical domain can be determined by inverting eqn (23) with appropriate $\widetilde{\mathbf{d}}_{0}, \tilde{\mathbf{s}}_{0}, \widetilde{\mathbf{d}}_{1}$ and $\tilde{\mathbf{s}}_{1}$ for the boundary conditions under consideration. The total field is obtained by adding the image field to that corresponding to eqn (16).

\section{ENERGETIC FORCE}

In this section the elastic fields derived in the previous section are used to study the image force on a dislocation shown in Fig. 1 and the interactive force between two like dislocations in a strip shown in Fig. 2. Numerical results are presented for W and K. Both crystals have $\mathrm{BCC}$ lattice structure, which is invariant in symmetry to $90^{\circ}$ rotations about the cubic axes. Only three elastic constants are independent for cubic crystals. The independent elastic constants with respect to the cubic axes, $c_{11}, c_{12}$ and $c_{44}$, in units of $10 \mathrm{GPa}$ for $\mathrm{W}$ are $52.1,20.1$ and 16.0, respectively; those for $\mathrm{K}$ are $0.457,0.374$ and 0.263 , respectively (Hirth and Lothe, 1982). The corresponding anisotropy ratio $A$, defined as $2 c_{44} /\left(c_{11}-c_{22}\right)$, indicates that $\mathrm{W}$ is isotropic with $A=1$ and $\mathrm{K}$ is highly anisotropic with $A=6.35$. In terms of the crystallographic notation, the slip direction or the direction of the Burgers vector in both materials is taken to be [111] and the slip plane in $\mathrm{W}$ is chosen to be (101) and that in $\mathrm{K}(3 \overline{2} \overline{1})$ (Hirth and Lothe, 1982). The dislocation is assumed to be pure edge so that the dislocation line is perpendicular to the slip direction as well as to the normal of the slip plane. In the numerical computations, the slip plane is considered to be inclined at an angle $\psi$ with respect to the $\left(x_{1}, x_{3}\right)$ plane of the strip. The Burgers vector of the edge dislocation is given by $\mathbf{b}=b(\cos \psi, \sin \psi, 0)$ and the dislocation line is oriented

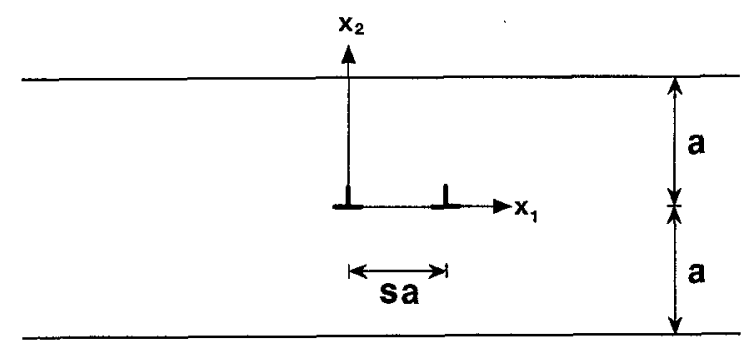

Fig. 2. Two like dislocations on the mid-plane of an elastic strip of thickness $2 a$. 
along the $x_{3}$ direction. It should be noted that although the Burgers vector is entirely in the $\left(x_{1}, x_{2}\right)$ plane, the displacement has a nonzero component in the $x_{3}$ direction for $\mathrm{K}$.

Consider first the image force on the dislocation in the strip. The image force per unit length is defined as the negative gradient of the interaction energy with respect to the position of the dislocation. Since the strip is infinite in the $x_{1}$ direction, the image force has a $x_{2}$ component only. The nonzero component $F_{\text {im }}$ can be calculated by the well-known Peach-Koehler formula (Peach and Koehler, 1950) as

$$
F_{\mathrm{im}}=-\left(\sigma_{11}^{(\mathrm{im})}(\eta, \psi) \cos \psi+\sigma_{12}^{(\mathrm{im})}(\eta, \psi) \sin \psi\right) \frac{b}{a}
$$

where $\sigma_{11}^{(\mathrm{im})}$ and $\sigma_{12}^{(\mathrm{im})}$ are the image stress components corresponding to $\phi^{(\mathrm{im})}$ of eqn (23) evaluated at the position of the dislocation. The image force can also be decomposed into a glide component of $F_{\mathrm{im}} \sin \psi$ in the slip direction and a climb component of $F_{\mathrm{im}} \cos \psi$.

The image force $F_{\mathrm{im}}$ as a function of the position of the dislocation for slip plane inclined at $\psi=0^{\circ}, 45^{\circ}$ and $90^{\circ}$ for $\mathrm{W}$ and $\mathrm{K}$ were computed under the three boundary conditions discussed in the previous section. It was found that if the forces were properly normalized, the results for $\mathrm{W}$ and $\mathrm{K}$ did not differ appreciably for all three boundary conditions. The normalization factor for free-free and free-fixed conditions $F_{\text {free }}$ is given by

$$
F_{\text {free }}=\frac{1}{4 \pi a} \mathbf{b}^{\mathrm{T}} \mathbf{L} \mathbf{b},
$$

where $\mathbf{L}$ is given by eqn (5) and that for fixed-fixed conditions $F_{\text {fix }}$ is given by

$$
F_{\text {fix }}=\frac{1}{4 \pi a} \mathbf{b}^{\mathrm{T}}\left(2 \mathbf{H}^{-1}-\mathbf{L}\right) \mathbf{b},
$$

where $\mathbf{H}$ is given by eqn (6). The factors $F_{\text {free }}$ and $F_{\text {fix }}$ are actually the forces in half-spaces with free and fixed surfaces, respectively, on a dislocation at a distance $a$ from the surfaces (Barnett and Lothe, 1974; Ting and Barnett, 1993). Either $F_{\text {free }}$ or $F_{\text {fix }}$ is invariant under rotation of the material with respect to the $x_{3}$ axis and hence is independent of the inclination angle $\psi$ (Ting and Barnett, 1993). The results for either W or K for free-free [case (1)], fixed-free [case (2)] and fixed-fixed [case (3)] boundary conditions are presented in Figs 3, 4 and 5, respectively. For free-free and free-fixed layers with $\psi=90^{\circ}$, the results agree well with those of Lee and Dundurs (1973). In fact, the expressions for the forces in these cases

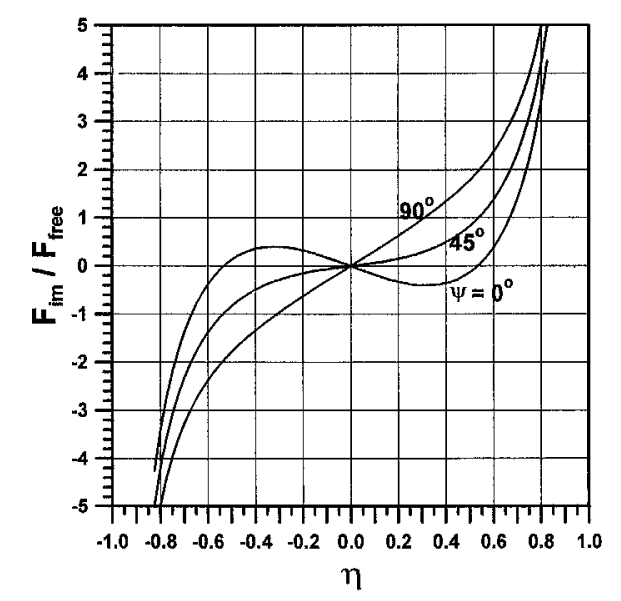

Fig. 3. Image force on an edge dislocation at $x_{2}=a \eta$ in a slip plane inclined at $\psi=0^{\circ}, 45^{\circ}$ and $90^{\circ}$, of a strip of thickness $2 a$ with free-free surfaces. 


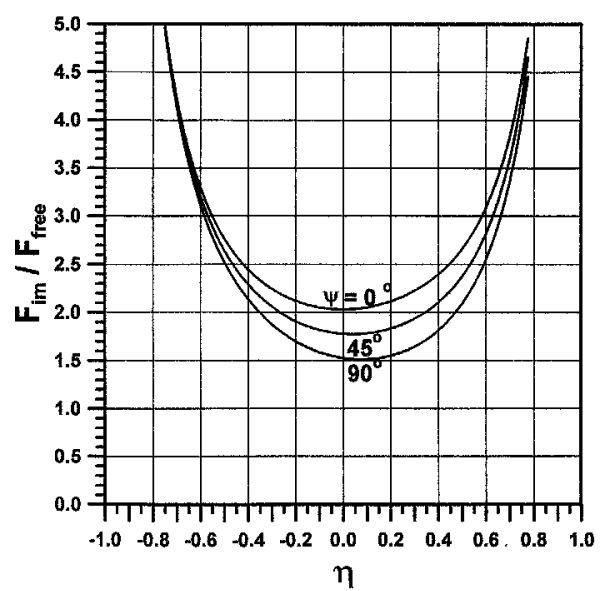

Fig. 4. Image force on an edge dislocation at $x_{2}=a \eta$ in a slip plane inclined at $\psi=0^{\circ}, 45^{\circ}$ and $90^{\circ}$, of a strip of thickness $2 a$ with fixed-free surfaces.

for isotropic material, in the form of inverse Fourier integrals, were checked to agree exactly with those derived by Lee and Dundurs.

In general the dislocation is attracted to the free surface and is repelled by the fixed surface. The magnitude of the image force on the dislocation at a given position increases with the slip plane inclination $\psi$ for free-free conditions but decreases for either fixed-fixed or fixed-free conditions. For free-free conditions, the origin is an unstable equilibrium position for $\psi=45^{\circ}$ and $90^{\circ}$. However, for $\psi=0^{\circ}$ the origin is a stable equilibrium position and the unstable equilibrium positions are shifted to $x_{2}= \pm 0.53$. For fixed-fixed conditions, the origin is always a stable position. No equilibrium positions exist for fixedfree conditions.

Consider next the interactive force of two like dislocations in the strip. For simplicity, the slip plane is assumed to coincide with the mid-plane of the strip with traction-free surfaces. A dislocation is located at the origin and the other is at $x_{1}=s a$ on the $x_{1}$ axis. The interactive force on the dislocation at $x_{1}=s a$ exerted by the other at the origin is given by

$$
F_{\mathrm{int}}=\sigma_{12}(s) \frac{b}{a}
$$

where $\sigma_{12}$ is the total stress component corresponding to $\phi$ of eqn (15). The interactive

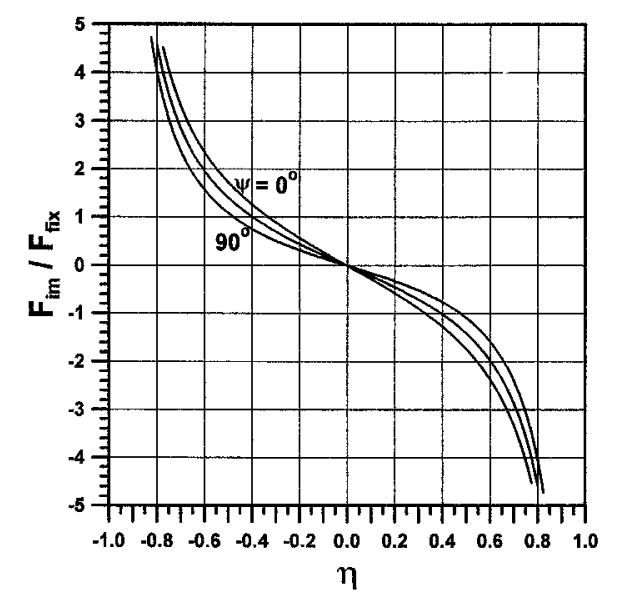

Fig. 5. Image force on an edge dislocation at $x_{2}=a \eta$ in a slip plane inclined at $\psi=0^{\circ}, 45^{\circ}$ and $90^{\circ}$, of a strip of thickness $2 a$ with fixed-fixed surfaces. 


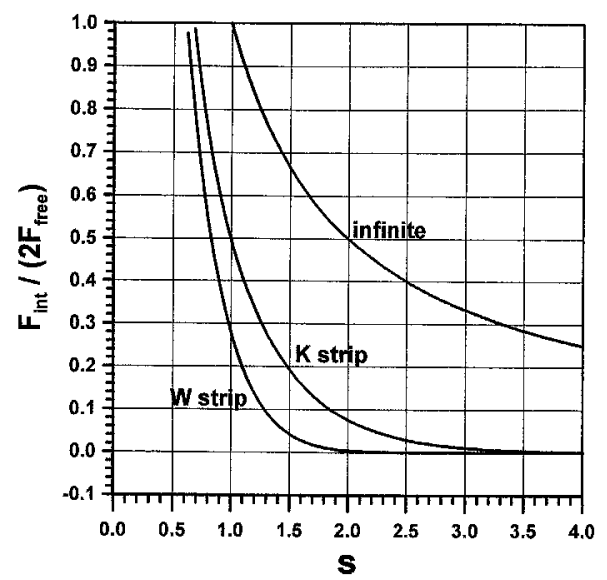

Fig. 6. Interactive force between two like dislocations separated at a distance $s a$ on the mid-plane of a strip with thickness $2 a$ with free-free surfaces. The slip plane coincides with the mid-plane of the strip.

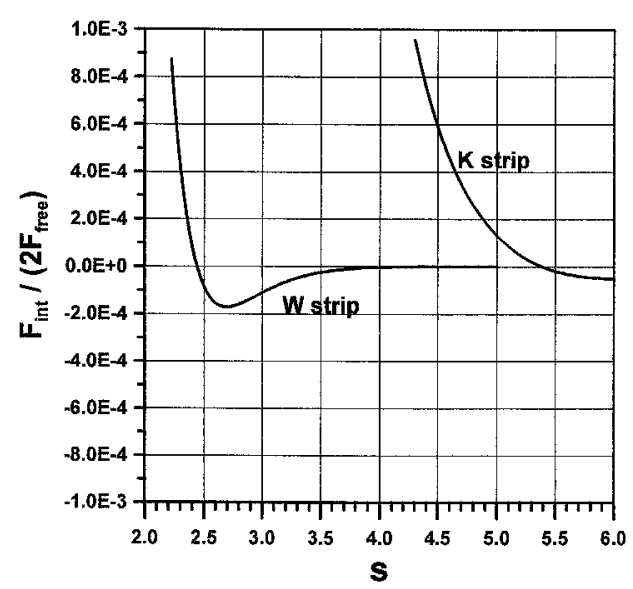

Fig. 7. Blown up plot of the curves for $\mathrm{W}$ and $\mathrm{K}$ in Fig. 6 for $s>2$.

force $F_{\text {int }}$ normalized by $2 F_{\text {free }}$ of the same material as a function of $s$ are plotted in Fig. 6 for $\mathrm{W}$ and $\mathrm{K}$. For comparison purposes, the normalized interactive force as the strip thickness is infinite, which varies as $1 / s$ for either crystal, is also plotted in Fig. 6 . Figure 6 shows that the presence of free surfaces reduces the forces significantly and that the reduction of the forces is more for isotropic $\mathrm{W}$ than for anisotropic $\mathrm{K}$ at a given separation $s$. For either material the forces fall off rapidly as $s$ increases. The forces, however, do not approach asymptotically to zero from the positive side. In fact it was shown by Nabarro and Kostlan (1978) for isotropic strips that the forces become negative or attractive beyond a certain separation. Indeed, if part of the curve for the W strip in Fig. 6 for $s>2$ is blown up as shown in Fig. 7, it is seen that the force becomes negative for $s>2.44$ for W. Thus for $\mathrm{W}$ a stable equilibrium separation of $2.44 a$ exists. A similar plot for the $\mathrm{K}$ strip, also given in Fig. 7, indicates that the same phenomenon also exists for the anisotropic crystal. The stable separation for $\mathrm{K}$, however, is $5.39 a$ which is more than twice as large as that for W. The existence of the stable separation between two similar dislocations in the strip implies that a set of dislocations lying in the mid-plane of the strip should form a regular array with spacing given by the critical separation.

\section{REFERENCES}

Barnett, D. M. and Lothe, J. (1974). An image force theorem for dislocations in bicrystals. J. Phys. 4, 1618-1635. Chou, Y. T. (1963). Planar stress field of a dislocation in an anisotropic plate. J. Appl. Phys. 34, 3608-3614.

Dundurs, J. and Sendeckyj, G. P. (1965). Behavior of an edge dislocation near bimetallic interface. J. Appl. Phys. 36, 3353-3354. 
Head, A. K. (1953). The interaction of dislocations and boundaries. Phil. Mag. 44, 92-94.

Hirth, J. P. and Lothe, J. (1982). Theory of Dislocations. 2nd edition, Wiley and Sons, New York.

Lee, M. S. and Dundurs, J. (1973). Edge dislocation in a surface layer. Int. J. Engng Sci. 11, 87-94.

Moss, W. C. and Hoover, W. G. (1978). Edge-dislocation displacements in an elastic strip. J. Appl. Phys. 49, $5449-5451$.

Nabarro, F. R. N. and Kostlan, E. J. (1978). The stress fields of a dislocation lying in a plate. J. Appl. Phys. 49, $5445-5448$.

Peach, M. and Koehler, J. S. (1950). The forces exerted on dislocations and the stress fields produced by them. Phys. Rev. 80, 436-439.

Stroh, A. N. (1958). Dislocations and cracks in anisotropic elasticity. Phil. Mag. 3, 625-646.

Stroh, A. N. (1962). Steady state problems in anisotropic elasticity. J. Math. Phys. 41, 77-103.

Ting, T. C. T. and Barnett, D. M. (1993). Image force on line dislocations in anisotropic elastic half-spaces with a fixed boundary. Int. J. Solids Structures 30(3), 313-323.

Wang, M. Z., Ting, T. C. T. and Yan, G. (1993). The anisotropic elastic semi-infinite strip. Q. Appl. Math. 51(2), 283-297.

Wu, K. C. (1991). Explicit crack-tip fields of an extending interface crack in an anisotropic bimaterial. Int. J. Solids Structures 37(4), 455-466. 